\title{
Diez años de apoyo a la Investigación Científica Básica por el CONACYT*
}

\author{
Luis Humberto Fabila Castillo**
}

\section{Resumen}

En este trabajo se revisan los resultados de diez años de apoyo a la investigación científica básica del CONACYT. Se identifican las áreas del conocimiento más fuertes en ciencia básica en México y se concluye que las instituciones en donde se realiza ciencia básica son principalmente las instituciones de educación superior públicas, seguidas de los centros de investigación también públicos, mientras que las instituciones de educación superior privadas y las empresas casi no realizan investigación en ciencia básica. Se destaca que la ciencia básica tuvo un crecimiento muy grande en las universidades estatales, alcanzando en los últimos años el nivel de las instituciones de educación superior del Distrito Federal. Finalmente, se ponen a consideración las implicaciones de estos hallazgos y de las políticas con las que se han otorgado estos apoyos.

\begin{abstract}
This paper reviews the results of ten years of support for basic scientific research by the CONACYT. The paper identifies the strongest areas of knowledge in basic science in Mexico and concludes that the institutions where basic science is done are mainly public higher education institutions, followed by also public research centers, while private institutions of higher education and companies carry out almost no research in basic science. Findings show that research on basic science in state universities has grown impressively in recent years, reaching the level of the institutions of higher education of the Federal District. Finally, the implications of these findings as well as the public policies through which support has been granted are discussed.
\end{abstract}

Palabras clave: ciencia básica, CONACYT, instituciones de educación superior públicas, instituciones de educación superior privadas, universidades estatales.

Key words: basic scientific research, CONACYT, public higher education institutions, private higher education institutions, state universities.

* Una parte de los datos que se presentan en éste artículo, fueron tomados del estudio "Evaluación de Impactos del Programa de Apoyos a Proyectos de Investigación de Ciencia Básica", elaborado por Esteva Maraboto Consultores, S.C. para el Consejo Nacional de Ciencia y Tecnología. Agradezco la revisión crítica que realizaron al trabajo, la QBP Guadalupe de los Ángeles $\mathrm{Cu}$ Tinoco y el Ing. José Antonio Esteva Maraboto.

* Luis Humberto Fabila Castillo, obtuvo el grado de Doctor en Ciencias con especialidad en Inmunología en la Escuela Nacional de Ciencias Biológicas del Instituto Politécnico Nacional, en donde es Profesor-Investigador. Actualmente tiene una licencia sin goce de sueldo para laborar como Director de Investigación Científica Básica en el Consejo Nacional de Ciencia y Tecnología de México. 
e considera que a nivel mundial, la mayor parte del conocimiento científico se genera en el sector académico y de investigación financiado por los gobiernos. Este conocimiento contribuye en forma muy importante al desarrollo tecnológico y económico de los países, impulsando la innovación (Stephan, 2001; OCDE, 2012). Existen numerosos ejemplos de cómo descubrimientos científicos básicos financiados por el sector público, han dado al paso de los años desarrollos prácticos de gran impacto tanto social como económico (Stephan, 2001). En el pasado se consideró que la ciencia básica alimentaba de forma directa a los procesos de innovación, pero actualmente se estima que estos últimos se enriquecen, además del conocimiento científico, de una combinación de fuentes y actores. Sin embargo, es claro que los productos y procesos nuevos que se desarrollan en las empresas, requieren de investigación académica reciente (Mansfield, 1991) y no hay duda de que la mayor parte de los productos innovadores tecnológicos con un alto valor añadido, tienen una gran cantidad de elementos derivados del conocimiento científico básico (Caraça y Mendoça, 2009). De ahí la importancia de que los países que deseen impulsar la innovación, tengan y desarrollen una buena ciencia básica.

En el caso de México, desde 1970 - año en el que fue creado el Consejo Nacional de Ciencia y Tecnología (CONACYT) - , se dio importancia al apoyo de la ciencia básica con fondos públicos, formalizándose esta decisión en 1975 al elaborarse el llamado "Programa Indicativo de Investigación sobre Ciencias Básicas", en el que se propusieron políticas para la ayuda y desarrollo de la ciencia básica en el país. Dichas políticas se materializaron en el hecho de que, durante los primeros diez años del CONACYT, el $25 \%$ del financiamiento otorgado a proyectos de investigación se destinó a ciencias básicas (Márquez, 1982).

A la fecha, el Fondo SEP-CONACYT ha emitido 10 convocatorias de investigación científica básica (una cada año) y en los archivos de la Dirección de Investigación Científica Básica del CONACYT, donde reside la Secretaría Técnica del Fondo, se tiene una relación muy detallada de los apoyos solicitados y otorgados, en cuanto a la institución solicitante, las áreas del conocimiento demandadas, las modalidades de apoyo solicitadas, entre otras cosas.

Desde su creación, el CONACYT ha apoyado constantemente proyectos de ciencia básica, sin embargo, en la literatura no se encuentran estudios que 
analicen a profundidad en qué instituciones se desarrolla la ciencia básica, cuáles son las áreas más dinámicas en el país, qué impactos han tenido estos apoyos o las políticas para otorgarlos. En el extenso libro de Márquez (1982) se analizan las políticas y acciones que tomó el CONACYT durante sus primeros diez años de creación y aunque una parte significativa habla de la ciencia básica, no se plantean las preguntas anteriores, además de que la información es de hace más e 30 años.

El objetivo de este trabajo es hacer un análisis descriptivo con información de las Convocatorias de Investigación Científica Básica que ha emitido el Fondo SEP-CONACYT desde su creación hace diez años. Es una ventana de oportunidad para contestar algunas de las preguntas planteadas anteriormente, viendo de primera mano qué efectos y resultados ha tenido el Fondo SEP-CONACYT en algunos aspectos del desarrollo de la ciencia básica en México, así como para revisar algunas de las políticas con las que se han otorgado.

Por esta razón, primero se describe qué áreas del conocimiento y modalidades de proyectos se apoyan y cómo se lleva a cabo el proceso de evaluación. En seguida, se analiza la manera en que han sido distribuidos los apoyos por áreas del conocimiento, modalidades y tipo de instituciones para finalmente discutir sobre la información que proporciona el análisis realizado.

\section{El fondo SEP - CONACYT}

La actualmente vigente Ley Orgánica del CONACYT, publicada el 5 de junio de 2002, indica en su artículo segundo que el CONACYT tendrá por objeto: "promover el desarrollo de la investigación científica y tecnológica, la innovación, el desarrollo y la modernización tecnológica del país". La fracción II del mismo artículo, destaca que para llevar a cabo lo anterior, al CONACYT le corresponderá realizar lo siguiente: "Apoyar la investigación científica básica y aplicada y la formación y consolidación de grupos de investigadores en todas las áreas del conocimiento, las que incluyen las ciencias exactas, naturales, de la salud, de humanidades y de la conducta, sociales, biotecnología y agropecuarias, así como el ramo de las ingenierías".

Con fundamento en la Ley de Ciencia y Tecnología en vigor y conforme a lo estipulado en la Ley Orgánica del CONACYT, la Secretaría de Educación Pública (SEP) y el CONACYT, constituyeron en diciembre de 2002, el fideicomiso denominado "Fondo Sectorial de Investigación para la Educación" -conocido comúnmente como Fondo SEP-CONACYT-, con 
el propósito fundamental de apoyar proyectos de investigación científica básica. El CONACYT nombra a un Secretario Técnico del Fondo, quien se encarga de la operación técnica del mismo (emisión de convocatorias, recepción de solicitudes, evaluación y seguimiento de los proyectos). En agosto de 2002 se emitió la convocatoria denominada "Proyectos de Investigación Científica Básica" que si bien antecedió por unos meses a la formalización del Fondo, ya menciona que los proyectos se financiarán con recursos provenientes del "Fondo Sectorial SEP-CONACYT". Un año después, en agosto de 2003, se emitió la primera convocatoria formal del Fondo, la cual fue denominada "Convocatoria de Investigación Científica Básica 2003".

Al amparo de la misma Ley de Ciencia y Tecnología, el CONACYT ha constituido hasta el año 2012 otros 19 Fondos Sectoriales, los cuales tienen como finalidad realizar investigación aplicada, desarrollo tecnológico o innovación en temas de interés para el sector con el que se constituye el Fondo (energía, salud, medio ambiente, etc.). Además, dentro del Fondo SEP-CONACYT se realiza investigación aplicada en temas relevantes para el sector educación. No obstante, del presupuesto total que ha manejado el Fondo, el $98 \%$ se ha dedicado a apoyar la investigación científica básica.

En todas las convocatorias para realizar investigación aplicada, los participantes envían una pre-propuesta, que pasa primero por un análisis de pertinencia, en el que tanto científicos como funcionarios del sector, revisan si las pre-propuestas responden y son pertinentes a las demandas sector. De ser así, se envía la propuesta completa para su evaluación final. En el caso de la Convocatoria de Investigación Científica Básica del Fondo SEP-CONACYT, no se hace un análisis de pertinencia, ya que no se trata de propuestas de investigación aplicada, desarrollo tecnológico o innovación que respondan a una demanda sectorial, sino de propuestas que buscan avanzar el conocimiento científico en prácticamente cualquier área del conocimiento. Debido a esta diferencia fundamental con los otros Fondos Sectoriales de CONACYT, en este trabajo solamente se analizan los resultados de las Convocatorias de Investigación Científica Básica del Fondo SEP-CONACYT.

Por otra parte, en el informe de labores 2012 del CONACYT (2012: 15) se indican los resultados de los Fondos sectoriales que emitieron convocatorias en dicho año. Se apoyaron 655 proyectos de Ciencia Básica por un monto de 937.1 millones de pesos, mientras que 12 Fondos sectoriales y las convocatorias de investigación aplicada del Fondo SEP-CONACYT, apoyaron 404 proyectos por un total de 802.6 millones de pesos. De acuerdo con estos datos, el apoyo a ciencia básica dentro de los Fondos sectoriales que maneja CONACYT, se llevó el 53.8\% del total del presupuesto 
que se concursó en 2012. Es importante mencionar que para estos cálculos no se tomó en cuenta la convocatoria de hidrocarburos de la Secretaría de Energía, mencionados en la misma fuente, debido a que son proyectos de orientación tecnológica y de gran aliento, que tienen presupuestos muy altos que no se comparan con el tipo de proyectos y financiamientos que otorgan los demás Fondos.

\section{Características de las convocatorias de Investigación Científica Básica}

De acuerdo a lo estipulado en las convocatorias emitidas, el Fondo SEPCONACYT tiene como propósito: “apoyar proyectos de investigación científica básica que generen conocimientos de frontera y contribuyan a mejorar la calidad de la educación superior y a la formación de científicos y académicos". Las convocatorias van dirigidas a "las Instituciones de Educación Superior (IES), Centros de Investigación públicos y privados y demás personas que se encuentren inscritos en el Registro Nacional de Instituciones y Empresas Científicas y Tecnológicas (RENIECYT)" (Bases de las Convocatorias de Investigación Científica Básica, s/p).

Las áreas del conocimiento en las que se apoyan proyectos son: I. FísicoMatemáticas y Ciencias de la Tierra, II. Biología y Química, III. Medicina y Ciencias de la Salud, IV. Humanidades y Ciencias de la Conducta, V. Ciencias Sociales y Economía, VI. Biotecnología y Ciencias Agropecuarias, VII. Ciencias de la Ingeniería y VIII. Investigación Multidisciplinaria. Del área I a la VII hay coincidencia con las áreas en las que está agrupado el Sistema Nacional de Investigadores del CONACYT. El área VIII, que se introdujo a partir de la convocatoria 2003, se abrió para incluir proyectos que implican la participación de especialistas de más de una de las áreas anteriores.

Por otra parte, en las convocatorias se han definido diferentes modalidades de proyectos, aplicables a todas las áreas del conocimiento. Algunas modalidades se han mantenido durante las 10 convocatorias, como es el caso de las siguientes y que se citan tal cual se publicaron en las convocatorias:

\footnotetext{
“Apoyo a iniciativas de investigador joven. Tiene como propósito respaldar iniciativas presentadas por aquellos profesores-investigadores que inician su carrera académica o científica en alguna institución inscrita en el RENIECYT. Las propuestas deberán ser presentadas por profesores-investigadores que sean menores de cuarenta años a la fecha del cierre de esta Convocatoria" (Términos de Referencia de las Convocatorias de Investigación Científica Básica, s/p)
} 
“Apoyo a iniciativas de profesor investigador. La finalidad primordial es apoyar iniciativas presentadas por profesores-investigadores consolidados, cuya producción científica y experiencia en formación de recursos humanos sea de reconocido prestigio nacional e internacional" (Términos de Referencia de las Convocatorias de Investigación Científica Básica, s/p)

Otras modalidades se mantuvieron durante unas convocatorias y después desaparecieron o se modificaron. Tal es el caso de:

\begin{abstract}
“Apoyo complementario para gastos de operación. Esta modalidad tiene como propósito el apoyar a profesores-investigadores, a través de propuestas de investigación institucionales, con recursos para gasto corriente complementarios a la infraestructura y los recursos aportados por la institución" (Términos de Referencia de las Convocatorias de Investigación Científica Básica, s/p).
\end{abstract}

Estos apoyos se otorgaron desde la convocatoria 2002 hasta la 2008 y se dejaron de otorgar a partir de la convocatoria 2009.

En todas las convocatorias se han implementado varias modalidades para apoyar el trabajo en grupos de investigación, aunque con diferentes variantes. A partir de la convocatoria de 2009 quedó como:

\footnotetext{
“Apoyo a iniciativas presentadas por cuerpos académicos o grupos de investigación. Esta modalidad tiene como propósito apoyar iniciativas de investigación que propicien o fortalezcan el trabajo en equipo y la producción académica conjunta de cuerpos académicos o de grupos de investigación" (Términos de Referencia de las Convocatorias de Investigación Científica Básica).
}

En las convocatorias anteriores a 2009 se distinguieron tres variantes de iniciativas presentadas por cuerpos académicos o grupos de investigación: a) iniciativas presentadas por un cuerpo académico o por un grupo de investigación, b) iniciativas presentadas por un cuerpo académico o grupo de investigación consolidado y uno en consolidación y c) iniciativas presentadas por redes de cuerpos académicos o grupos de investigación consolidados. No hay registros que indiquen por qué se modificaron estas variantes. A partir de 2009 se incluyó la variante: iniciativas presentadas por cuerpos académicos o grupos de investigación de colaboración México-Centroamérica, sin que tampoco existan registros de qué motivó este cambio. Para el análisis que se hace en este trabajo, todas estas variantes se agruparon como "Grupos de Investigación". 
Las propuestas siempre han sido evaluadas por el sistema de revisión de pares académicos (peer review en inglés), a través de las llamadas comisiones de expertos que, en principio, han sido al menos una por cada área del conocimiento mencionadas anteriormente (ocho comisiones). Estas comisiones están conformadas por entre 5 y 20 investigadores, dependiendo del número de propuestas recibidas por área del conocimiento, de entre los cuales se nombra a un presidente para cada comisión. Sin embargo, debido al alto número de propuestas que llegan para algunas áreas, con frecuencia se forman más comisiones. Generalmente para el área I se conforma una sola comisión de física y matemáticas y otra de ciencias de la tierra. Para el área II se forma una comisión de biología y otra de química. Para dictaminar las propuestas, las comisiones de expertos se apoyan en la opinión de peritos en el tema de cada propuesta, quienes sirven como árbitros. Estos árbitros forman parte del Registro CONACYT de Evaluadores Acreditados (RCEA) y son seleccionados por los mismos miembros de la comisión. Esta parte del proceso de evaluación se realiza mediante un sistema informático vía internet. Posteriormente las comisiones de expertos sesionan en pleno y, tomando como base las opiniones de los árbitros, dictaminan cada una de las propuestas en forma colegiada. Finalmente se conforma una Comisión de Evaluación, presidida por el Secretario Técnico del Fondo y conformada por los presidentes de cada una de las comisiones de expertos la que, partiendo de los dictámenes de las comisiones de expertos y un estimado del presupuesto disponible, elabora una lista de propuestas para ser apoyadas que se presenta al Comité Técnico y de Administración del Fondo para su aprobación final.

El Fondo ha tenido desde su creación cuatro Secretarios Técnicos, incluyendo al autor del presente trabajo, quien se ha entrevistado con los tres Secretarios anteriores y todos han señalado que en todas las convocatorias, la Comisión de Evaluación ha distribuido el presupuesto entre las áreas del conocimiento en forma proporcional a la demanda y a la proporción de proyectos aprobados. Lo que significa que no se favorece algún área del conocimiento en particular.

De acuerdo a la convocatoria, las propuestas deben dejar explícita la importancia de la investigación en la generación de conocimiento científico básico y este es un punto que evalúan tanto los árbitros como las comisiones de expertos. Una propuesta puede rechazarse porque la comisión dictamine que no es de ciencia básica, independientemente de su calidad 
científica. En general, cuando la respuesta a la pregunta central planteada en la propuesta, hace avanzar el conocimiento del área sin considerar una aplicación inmediata, se considera que es de ciencia básica. Sin embargo, el investigador que propone el proyecto puede dejar en claro que la respuesta a la pregunta central que se plantea, abre posibilidades aplicativas en futuros proyectos y esto es considerado aceptable. De cualquier forma, dadas las particularidades de cada área del conocimiento, la decisión final en cuanto a que si un proyecto es considerado de ciencia básica, se ha dejado al criterio colegiado de cada una de las comisiones de expertos.

\section{Resultados}

Proyectos solicitados y aprobados por área del conocimiento

Durante el período de estudio se recibieron 21,317 propuestas de proyectos. Como puede observarse en la tabla 1, la mayoría de las propuestas correspondieron al área de biología y química (21.4\%), seguida muy de cerca del área de ciencias de la ingeniería (19.3\%). En las áreas de: físico-matemáticas y ciencias de la tierra; biotecnología y ciencias agropecuarias; y de medicina y ciencias de la salud se recibieron entre el 13.5\% y el 16.7\% del total de propuestas. Las áreas que menos propuestas recibieron fueron las de ciencias sociales y economía; humanidades y ciencias de la conducta; y de investigación multidisciplinaria (entre 3.5\% y 6.4\%).

Tabla 1. Proyectos solicitados y aprobados en el período 2002-2011 por área de conocimiento

\begin{tabular}{|c|c|c|c|c|}
\hline Área & $\begin{array}{l}\text { No. de Proyectos } \\
\text { Solicitados }\end{array}$ & $\%$ del Total & $\begin{array}{c}\text { No. de Proyectos } \\
\text { Aprobados }\end{array}$ & $\begin{array}{c}\% \text { de Proyectos } \\
\text { Aprobados }\end{array}$ \\
\hline Biología y química & 4,563 & $021.4 \%$ & 1,553 & $34.0 \%$ \\
\hline Ciencias de la ingeniería & 4,116 & $019.3 \%$ & 1,327 & $32.2 \%$ \\
\hline $\begin{array}{l}\text { Físico-matemáticas y ciencias } \\
\text { de la tierra }\end{array}$ & 3,565 & $016.7 \%$ & 1,585 & $44.4 \%$ \\
\hline Medicina y ciencias de la salud & 2,897 & $013.5 \%$ & 0,854 & $29.4 \%$ \\
\hline Ciencias sociales y economía & 1,377 & $006.4 \%$ & 424 & $30.8 \%$ \\
\hline $\begin{array}{l}\text { Humanidades y ciencias de la } \\
\text { conducta }\end{array}$ & 1,096 & $005.1 \%$ & 417 & $38.0 \%$ \\
\hline Investigación multidisciplinaria & 761 & $003.5 \%$ & 257 & $33.7 \%$ \\
\hline TOTALES: & 21,317 & $100.0 \%$ & 7,225 & $33.8 \%$ \\
\hline
\end{tabular}

Fuente: elaboración del autor. 
De todas estas propuestas, se aprobaron para apoyo financiero un total de 7,225 proyectos, lo cual da un porcentaje global de aprobación del $33.8 \%$. Al analizar por área del conocimiento, el porcentaje de aprobación no varió mucho. Las dos áreas con más bajo porcentaje de aprobación fueron biotecnología y ciencias agropecuarias $(27.4 \%)$ y medicina y ciencias de la salud (29.4\%), mientras que el área de físico matemáticas y ciencias de la tierra fue la más alta (44.4\%). El resto de las áreas se sitúa no más de 4 puntos porcentuales por arriba o por debajo del $33.8 \%$, que es el promedio de todas las áreas (tabla 1).

Proyectos solicitados y aprobados por modalidad

En la tabla 2 se muestra el total de proyectos recibidos y aprobados por modalidad. En la modalidad de profesor investigador fue en la que más proyectos se recibieron (42.6\%), seguida de la de investigador joven $(27.2 \%)$. En las modalidades de grupos de investigación y de gastos de operación se recibieron menos proyectos ( $16.7 \%$ y $13.4 \%$, respectivamente).

Tabla 2. Proyectos solicitados y aprobados en el período 2002-2011 por modalidad

\begin{tabular}{|c|c|c|c|c|}
\hline Modalidad & $\begin{array}{c}\text { No. de Proyectos } \\
\text { Solicitados }\end{array}$ & \% del Total & $\begin{array}{c}\text { No. de } \\
\text { Proyectos } \\
\text { Aprobados }\end{array}$ & $\begin{array}{c}\text { \% de Proyectos } \\
\text { Aprobados }\end{array}$ \\
\hline Profesor investigador & 9,092 & $42.6 \%$ & 3,329 & $36.6 \%$ \\
\hline Investigador joven & 5,803 & $27.2 \%$ & 1,895 & $32.6 \%$ \\
\hline Grupos de investigación & 3,557 & $16.7 \%$ & 1,048 & $29.4 \%$ \\
\hline Gastos de operación & 2,865 & $13.4 \%$ & 953 & $33.2 \%$ \\
\hline TOTALES & 21,317 & $100 \%$ & 7,225 & $33.8 \%$ \\
\hline
\end{tabular}

Fuente: elaboración del autor.

Como ya se mencionó, el porcentaje global de proyectos apoyados fue de $33.8 \%$ y dicho porcentaje fue muy similar entre cada una de las diferentes modalidades, variando entre $29.4 \%$ para grupos de investigación y $36.6 \%$ para profesor investigador.

La distribución de proyectos aprobados por modalidad varió significativamente entre las diferentes convocatorias, tal cual se muestra en la gráfica 1.

Se observa que, con excepción de 2004, la mayoría de los proyectos apoyados pertenecen a la modalidad de Profesor Investigador. En el caso de los apoyos para Gastos de Operación, esta modalidad no estaba dirigida 
en particular a profesores investigadores o a jóvenes investigadores, probablemente la mayoría de estos apoyos se otorgaron a investigadores con el perfil de profesor investigador, ya que en el caso de los investigadores jóvenes en la misma convocatoria existió una modalidad específica para ellos.

De hecho esto explicaría por qué cuando se dejaron de otorgar los apoyos para gastos de operación (a partir de la convocatoria 2009) se elevó la proporción de apoyos para los profesores investigadores (gráfica 1).

\section{Gráfica 1. Distribución de los proyectos apoyados por modalidad (2002-2011)}

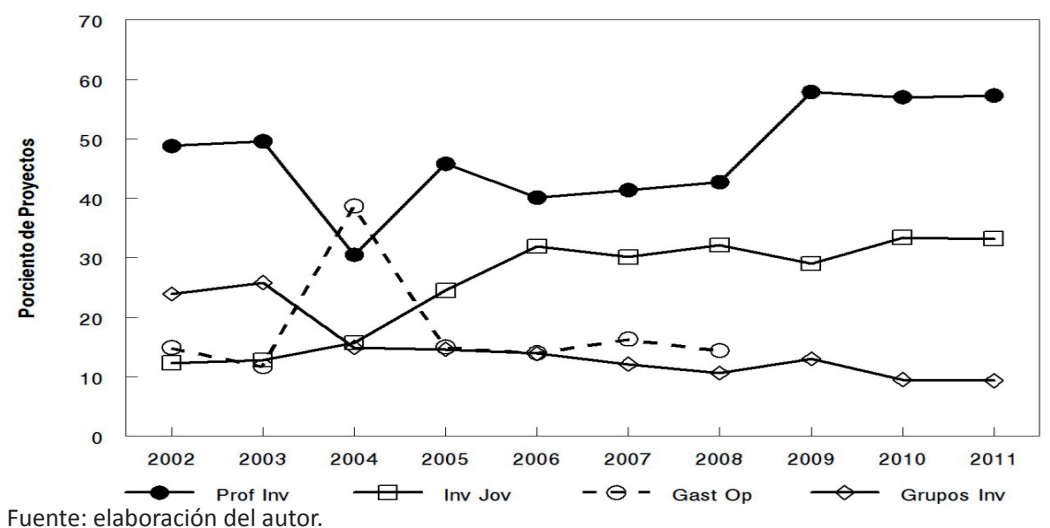

En el caso de los apoyos a Grupos de Investigación se observa que al inicio del Programa se apoyó un porcentaje significativo de proyectos en esta modalidad (más del 20\%), sin embargo, a partir de la convocatoria 2004 comenzó a disminuir y esta tendencia se ha mantenido hasta la convocatoria 2011, en la que se tuvo la participación más baja (9.3\%). Los apoyos en la modalidad de joven investigador muestran una tendencia interesante: en 2002 y 2003 se situaron en un poco más del 10\% de los proyectos apoyados, a partir de 2004 se comenzó a incrementar el porcentaje de proyectos apoyados, para situarse en alrededor del $30 \%$ en 2006 y se mantuvo alrededor de este valor de 2006 a 2011.

Proyectos apoyados por tipo de institución

Las instituciones a las que se les apoyaron proyectos de investigación se agruparon, por un lado, en Instituciones de Educación Superior públicas (IES Públicas), que comprenden a: la Universidad Nacional Autónoma de México (UNAM), el Instituto Politécnico Nacional (IPN), el Centro de Investigación y Estudios Avanzados del IPN, la Universidad Autónoma 
Metropolitana y las universidades estatales; Instituciones de Educación Superior privadas (IES Privadas), centros de investigación que pertenecen al CONACYT (Centros CONACYT) y por el otro, en centros de investigación o instituciones donde se realiza investigación y que dependen de alguna Secretaría de Estado (Salud, Energía, Agricultura, etc.), por ejemplo: hospitales de la Secretaría de Salud, hospitales o centros del Instituto Mexicano del Seguro Social, el Instituto Mexicano del Petróleo, etc. En términos generales, a este grupo se le denomina: "Centros de Investigación de Sectores Aplicativos". Un último grupo es el denominado "Empresas y otros", que incluye empresas o asociaciones civiles que están registradas en el RENIECYT y que han obtenido apoyos para proyectos de ciencia básica. La distribución de proyectos por tipo de institución, se muestra en la gráfica 2.

Es evidente que la mayoría de los proyectos se llevan a cabo en las IES públicas (71.4\%), seguidas por los Centros CONACYT (15.9\%) y los Centros de Investigación de los Sectores Aplicativos (10.2\%). Estos tres grupos que son financiados por el sector público, obtuvieron el $97.5 \%$ de los apoyos a investigación científica básica. La participación del sector privado es muy baja. Las IES privadas obtuvieron el $2 \%$ de los proyectos y las empresas y otras entidades el $0.5 \%$.

Gráfica 2. Distribución de proyectos aprobados por tipo de institución (2002-2011).

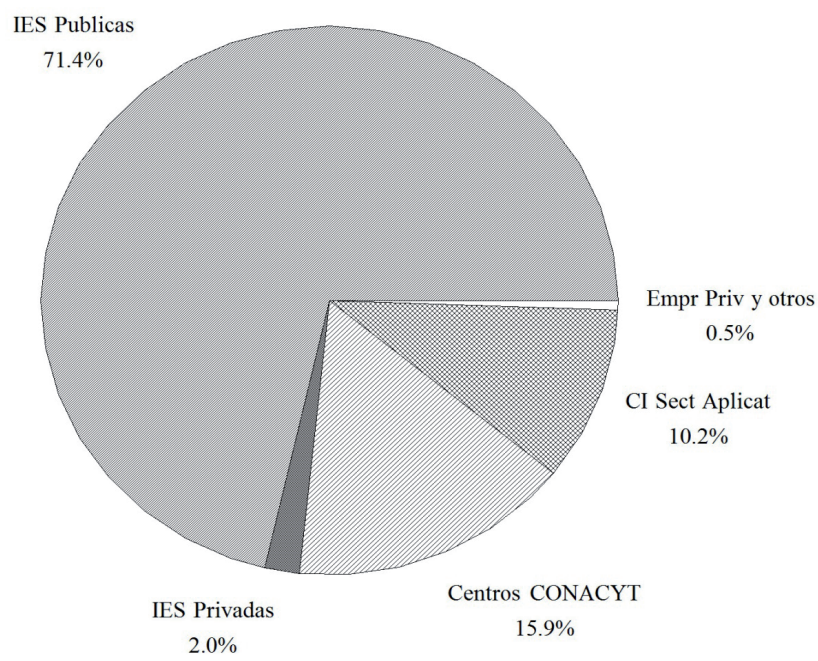

Fuente: elaboración del autor. 
Participación de las instituciones del Distrito Federal y de los estados de la República

Se analizó cómo se distribuyeron los proyectos entre las instituciones que se encuentran en la Ciudad de México (Distrito Federal) y las que se encuentran en los Estados de la República, independientemente del tipo de institución. La UNAM, el IPN y el CINVESTAV tienen unidades académicas y centros de investigación en varios estados de la República, que han sido creados para colaborar con la descentralización de las actividades científicas. Los proyectos asignados a estos centros y unidades, fueron contabilizados dentro de los asignados a los Estados. En la gráfica 3, se observa que los apoyos a ciencia básica muestran una clara tendencia a otorgarse en mayor proporción a instituciones de los estados de la república. En 2002 el $52.2 \%$ de los apoyos se otorgaron a instituciones de los estados y esta cifra se incrementó en 2003 y 2004, para después volver a disminuir en 2005 y 2006. A partir de 2007, se nota una tendencia sostenida de incremento a los proyectos de ciencia básica en los Estados, situándose en alrededor del $60 \%$ entre 2008 y 2011.

\section{Gráfica 3. Proyectos apoyados en el Distrito Federal y en los estados de la República entre 2002 y 2011}

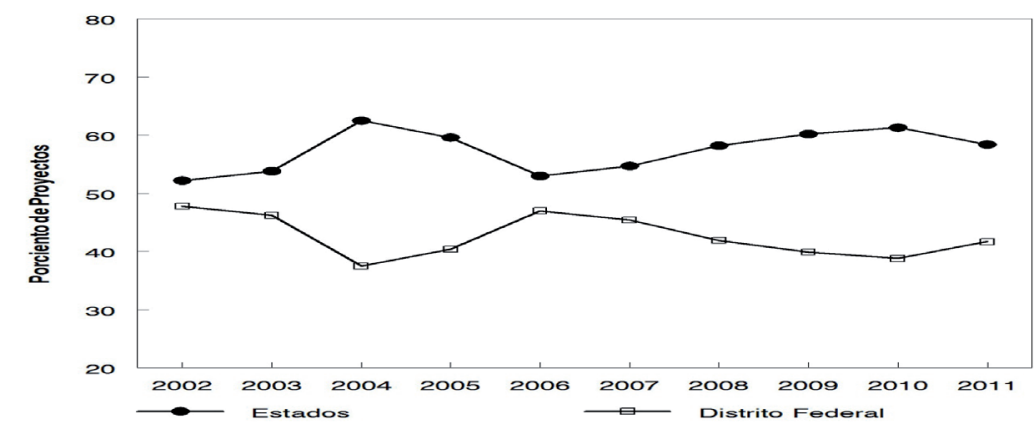

Fuente: elaboración del autor.

Por otra parte, dado que la mayoría de los proyectos de ciencia básica se desarrollan en las Instituciones de Educación Superior públicas (gráfica 2), se analizó dentro de estas instituciones, cómo se han distribuido los proyectos entre las instituciones que se encuentran en el Distrito Federal y las que se encuentran en los estados de la República. Para lograrlo se agruparon a las instituciones participantes en, por una parte, aquellas que son públicas y que se encuentran en el Distrito Federal: la Universidad Nacional 
Autónoma de México (UNAM); el Centro de Investigación y de Estudios Avanzados del IPN (CINVESTAV); el Instituto Politécnico Nacional (IPN); y la Universidad Autónoma Metropolitana (UAM) (denominadas "IES D.F."). En el caso de la UNAM, el CINVESTAV y el IPN, se excluyeron los proyectos otorgados a las unidades localizadas fuera del D.F. y, por la otra, en otro grupo formado con las universidades estatales. Finalmente se estableció un tercer grupo conformado por el Sistema Tecnológico de la Secretaría de Educación Pública, que incluye a los Institutos Tecnológicos Regionales y Estatales, las Universidades Politécnicas y las Universidades Tecnológicas del Sistema de Universidades Estatales de Oaxaca. En la gráfica 4 se muestra la participación de estos tres grupos de instituciones entre 2002 y 2011, expresándola como porcentaje del total de proyectos asignados por convocatoria.

\section{Gráfica 4. Proyectos aprobados a las Instituciones de Educación Superior del} Distrito Federal y de los estados de la República.

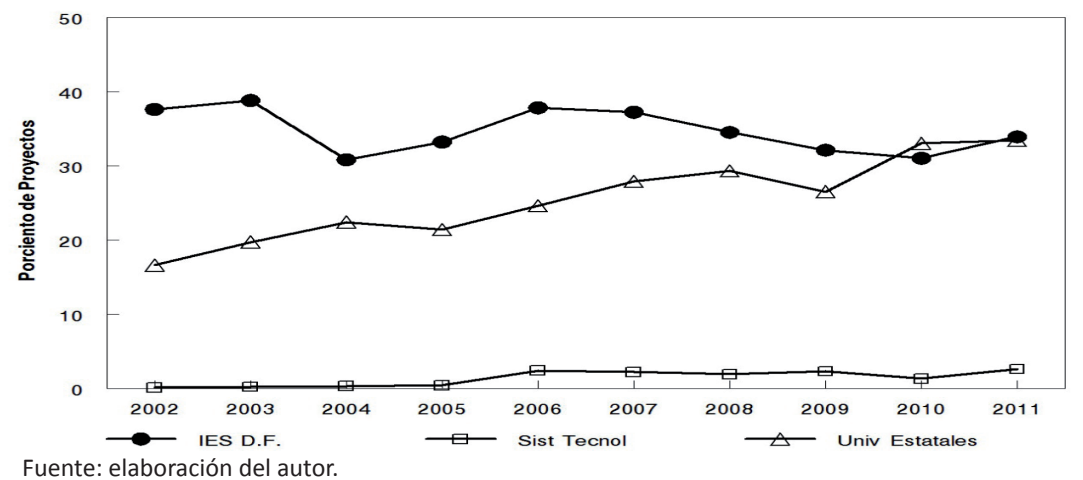

Para las IES del Distrito Federal, se nota una tendencia a que disminuya su participación, mientras que para las universidades estatales se nota una tendencia constante a aumentarla, pasando de un $16.6 \%$ de proyectos en 2002, a un 33.4\% en 2011. De hecho en 2010 los apoyos para proyectos en las universidades estatales superaron al de las IES del Distrito federal y en 2011 fueron prácticamente iguales.

Es claro que ha habido una disminución constante de la participación de las Instituciones del Distrito Federal pues, mientras que en 2002 obtuvieron el 51\% de los proyectos, en 2011 esta participación bajó al 39.3\%. Esta disminución es explicada por un incremento constante de la participación de las universidades estatales. Por otra parte, el Sistema Tecnológico de la SEP tuvo una participación mínima entre 2002 y 2005 y aunque a partir 
de 2006 su participación ha aumentado un poco, no se nota una tendencia clara al alza.

Las universidades estatales con mayor participación

Dado que el incremento de la participación de las instituciones de los estados se debe principalmente a las universidades estatales, se analizó la participación de cada una de ellas. En la tabla 3 se muestran las diez universidades estatales que han obtenido el mayor número de proyectos en el período de estudio.

Tabla 3. Las diez universidades estatales que han obtenido mayor número de proyectos de ciencia básica entre 2002 y 2011.

\begin{tabular}{|c|c|c|c|}
\hline No & UNIVERSIDAD & No de Proyectos & \% del Total ${ }^{1}$ \\
\hline 1 & Universidad Autónoma de Nuevo León & 211 & $2.9 \%$ \\
\hline 2 & Universidad Autónoma de San Luis Potosí & 182 & $2.5 \%$ \\
\hline 3 & Universidad de Guadalajara & 151 & $2.1 \%$ \\
\hline 4 & Universidad Michoacana de San Nicolás de & 148 & $2.0 \%$ \\
\hline 5 & Hidalgo & 146 & $2.0 \%$ \\
\hline 6 & Universidad Autónoma del Estado de Morelos & 140 & $1.9 \%$ \\
\hline 7 & Universidad de Guanajuato & 108 & $1.5 \%$ \\
\hline 8 & Universidad de Sonora & 99 & $1.4 \%$ \\
\hline 9 & Universidad Veracruzana & 80 & $1.1 \%$ \\
\hline 10 & Universidad Autónoma de Baja California & 76 & $1.0 \%$ \\
\hline & Tutal: & 1341 & $18.8 \%$ \\
\hline
\end{tabular}

Estas diez Universidades se encuentran distribuidas principalmente en la zona norte y centro del país y llama la atención que ninguna de las universidades de los estados del sur aparezca entre las diez primeras, aunque hay que mencionar que la universidad que ocupa el lugar número 11 es la Universidad Autónoma de Yucatán, la cual ha participado con 74 proyectos, sólo dos menos que la Universidad Autónoma de Baja California que ocupa el décimo lugar. Hay que tomar en cuenta que la participación de las universidades que se encuentran entre los primeros seis lugares es relativamente homogénea, es decir, no hay un predominio muy marcado de una o dos universidades en particular, lo que implica que el desarrollo de 
la ciencia básica en el Sistema de Universidades Estatales no es explicable por un desarrollo muy grande de unas pocas instituciones. Es importante mencionar que las diez Universidades con mayor participación obtuvieron el $72.3 \%$ del total de proyectos obtenidos por todas las universidades estatales, por lo que entre las 21 universidades estatales restantes se distribuye sólo el $27.7 \%$ de los proyectos obtenidos por este grupo de instituciones.

\section{Discusión}

El análisis de los datos derivados de diez años de apoyo a la investigación científica básica por parte del CONACYT, nos permite tener una "radiografía" de cómo se ha desarrollado la ciencia básica en el país durante los últimos años y cuál es su situación actual.

La demanda de apoyos al Programa de Ciencia Básica es muy alta, sumando 21,317 propuestas dentro del período estudiado. De estas propuestas, se apoyaron 7,225 proyectos, es decir, el $33.8 \%$.

$\mathrm{Al}$ analizar el porcentaje de proyectos apoyados por área, en relación a su demanda, vemos que la única área que se aleja un poco más de la media de aprobación (33.8\%), es la de físico matemáticas y ciencias de la tierra, que está 10.6 puntos porcentuales por arriba de la media. El resto de las áreas no se alejan más de 6.5 puntos de la media. Esto es importante, porque indica que las diferentes áreas tienen criterios de aceptación o rechazo de proyectos que no varían mucho entre ellas. Por lo tanto, la distribución de proyectos apoyados por área del conocimiento, nos indica cómo se distribuyen las fortalezas en ciencia básica en México entre las diferentes áreas del conocimiento.

De acuerdo a la tabla 1 se pueden identificar dos bloques fuertes, uno compuesto por las llamadas ciencias "duras" (física, matemáticas y química) y de las ciencias de la vida (biología), que en conjunto representan el $43.4 \%$ de los proyectos apoyados. El otro bloque está compuesto por las áreas que podríamos considerar más aplicativas, como son medicina, biotecnología y ciencias de la ingeniería, que en conjunto representan el $41.4 \%$. Las áreas relacionadas con las ciencias sociales (humanidades, ciencias de la conducta, ciencias sociales y economía) tienen una participación menor, ya que suman sólo el $11.6 \%$.

Como ya se trató en la sección correspondiente al sistema de evaluación, esta distribución no explica el por qué se aplicó una política de un mayor o menor apoyo a un área del conocimiento en particular. Por lo tanto, se puede considerar que la tabla 1 nos da únicamente una idea aproximada 
del tamaño de las capacidades para poder realizar investigación básica en el país, en cada una de las áreas mostradas. Para tener un panorama más completo de las mismas, haría falta analizar la habilidad para obtener fondos para la investigación de fuentes distintas al CONACYT, tanto nacionales como internacionales.

Sin embargo, un punto de reflexión apunta a detenerse en qué efectos tiene esta política en el desarrollo científico del país. En la forma como se ha manejado hasta ahora, con lo que se cuenta es un mecanismo que permite apoyar desde el CONACYT, a la ciencia básica que se va desarrollando de manera "natural" en el país, de tal manera que las instituciones que hacen investigación puedan hacer planes para el impulso a las áreas de investigación básica que consideren adecuadas, sabiendo que de parte de CONACYT existen mecanismos para su apoyo.

Otro posible enfoque sería que el país definiera prioridades de desarrollo científico en algunas áreas del conocimiento, las cuales para mantenerse en las fronteras del conocimiento, necesitarían promover el desarrollo de ciencia básica en áreas y temas más focalizados. En este escenario, la política a implementar sería la de apoyar la investigación básica dándole cierta preferencia a estas áreas o temas. Un esquema de este tipo tiene la ventaja de articular a la ciencia básica con las prioridades de desarrollo del país, aunque tiene la desventaja de inhibir la creatividad de los investigadores en las áreas no apoyadas o con apoyos menores. Para tomar una decisión así sería recomendable analizar las experiencias y resultados obtenidos por otros países.

Las investigaciones multidisciplinarias merecen un comentario aparte. De hecho, no se trata de un área de conocimiento en sí misma, sino de una forma de trabajo que en principio, debería dar mejores resultados al atacar un problema de investigación desde diferentes enfoques. La proporción de proyectos que se han otorgado en esta modalidad es muy baja (3.5\%) y una posible explicación es que los investigadores que realizan investigación básica en el país, continúan realizando sus investigaciones desde un punto de vista unidisciplinario.

Otra explicación posible es que los estímulos para presentar propuestas multidisciplinarias, no sean lo suficientemente atractivos para los investigadores. El estímulo en las diversas convocatorias ha sido ofrecer una mayor cantidad de recursos (alrededor del doble) para los proyectos multidisciplinarios, en relación a los proyectos individuales. Esto quiere decir que si en el proyecto participan dos investigadores diferentes, los recursos asignados serían similares a los que obtendría cada investigador por sepa- 
rado. Sin embargo, si participan tres investigadores, los recursos para cada investigador serían menores a los que obtendría cada uno por separado.

Probablemente, esto es un factor que desalienta la participación en este tipo de proyectos. De acuerdo a esto, una forma de promover los proyectos multidisciplinarios, sería la de ofrecer una cantidad sensiblemente mayor de recursos para estos proyectos.

Otro punto que es necesario revisar es el de las convocatorias, debido a que no se define claramente qué proyectos se consideran multidisciplinarios. Lo que se ha estipulado es que son: "propuestas formuladas en aspectos básicos en donde se note claramente la participación y división de las diferentes áreas o disciplinas del conocimiento en la solución de un proyecto de investigación que por su complejidad, no pueda resolverlo una disciplina individualmente" (Términos de Referencia de las Convocatorias de Investigación Científica Básica, s/p). La interpretación de lo que es una propuesta de investigación multidisciplinaria se ha dejado a juicio de las Comisiones de Expertos. Una definición más clara en las convocatorias, aunada a la posibilidad de obtener una cantidad atractiva de recursos, podría incrementar la participación de este tipo de proyectos.

En relación con los apoyos para las diferentes modalidades, hay varios puntos a resaltar. De acuerdo con las convocatorias cada modalidad tiene un objetivo diferente. Se puede interpretar que los apoyos a profesores investigadores están orientados a darles elementos a los investigadores ya consolidados para que puedan realizar investigación de frontera y de calidad internacional, mientras que los apoyos a investigadores jóvenes están orientados a darles elementos para que puedan consolidarse y alcanzar en poco tiempo un nivel también internacional. Por su parte, los apoyos a grupos de investigación buscan promover el trabajo interinstitucional en equipo y finalmente, los apoyos a gastos de operación tienen como propósito dar apoyos a investigadores en instituciones que tienen una buena infraestructura y no requieren apoyo para compra de equipo. No hay registros del por qué se dejaron de otorgar apoyos en esta última modalidad a partir de 2009. Una posible explicación es que debido a problemas presupuestales, aún las instituciones bien equipadas comenzaron a tener problemas para mantener su infraestructura, por lo que la proporción de proyectos otorgados en esta modalidad comenzó a decaer (gráfica 1).

La proporción de proyectos aprobados con relación a los solicitados, fue muy parecida entre las diferentes modalidades (tabla 2), sin embargo, ha habido variaciones importantes en la distribución de proyectos entre las diferentes convocatorias. En la gráfica 1 observamos que a partir de 2004, la proporción de proyectos otorgados en la modalidad de grupos de 
investigación comenzó a disminuir en forma constante, quedando en 2011 con una participación de sólo el 9.3\% de los proyectos aprobados, cuando al inicio del programa llegó a tener una participación de casi 25\%. Las comisiones de evaluación han sido cada vez más exigentes en considerar lo que es un proyecto de grupos de investigación y en las últimas convocatorias (2009 a la 2011), han acordado que para considerar un proyecto como de grupo de investigación, debe de haber participación de investigadores de instituciones diferentes, o si son de la misma institución, de unidades académicas distintas. Los apoyos a investigadores jóvenes merecen comentarios aparte. En la gráfica 1 se observa que los apoyos en esta modalidad, muestran un incremento importante y constante a partir de 2002. En este año, el $12.3 \%$ de los proyectos otorgados fueron en esta modalidad. Para 2011 esta participación subió al 33\%, lo que significa un crecimiento de casi el triple. Esto se explica por un incremento en la demanda; en 2002 el 12,4\% de las proyectos solicitados era en la modalidad de investigador joven y se fue incrementando año con año, para llegar a una demanda de $34.5 \%$ de los proyectos solicitados en 2011, mientras que los porcentajes de aprobación no variaron sensiblemente entre los diferentes años (datos no mostrados).

Como se ha comentado, los apoyos para todas las áreas y modalidades, se han dado en proporción a la demanda y a los porcentajes de aprobación, de tal forma que el incremento en apoyos para investigadores jóvenes no es el resultado directo de una política de apoyo a los jóvenes de parte del CONACYT, sino el resultado de un incremento en la demanda. Este incremento pudiera ser explicable por las políticas de contratación de investigadores jóvenes en las diferentes instituciones de educación superior, principalmente en las estatales y en parte en los Centros de Investigación. Los apoyos a la investigación científica básica del CONACYT, han jugado un papel importante en la consolidación de investigadores jóvenes y como veremos más adelante, han ayudado a la consolidación y crecimiento de la investigación en las universidades estatales. De hecho, alrededor del 40\% de los proyectos en modalidad de investigador joven, se han otorgado a universidades estatales.

Por otra parte, el análisis de los datos apunta a que son las Instituciones de Educación Superior públicas las que realizan la mayor parte de proyectos de investigación científica básica, seguida por los centros CONACYT y en tercer lugar los Centros de Investigación de los Sectores Aplicativos. Todas estas instituciones y centros pertenecen al gobierno y en su conjunto realizaron el $97.5 \%$ de los proyectos de investigación científica básica. La participación de instituciones de educación superior privadas y de empresas es mínima, con sólo el 2.5\% de los proyectos. Esto concuerda con 
los estudios que ha publicado la Organización para la Cooperación y el Desarrollo Económico (OCDE) en los que se encuentra que en la mayor parte de los países miembros de la OCDE, la investigación básica se realiza principalmente en las instituciones de educación y centros de investigación gubernamentales (OCDE, 2012). La poca participación de las instituciones de educación superior privadas y las empresas, muy probablemente se debe a que dentro de sus planes de desarrollo, no se contempla el realizar investigación y en los casos en que la investigación forme parte de sus planes de desarrollo, se privilegie más la investigación aplicada que la investigación básica. Como ya se comentó, el CONACYT tiene convocatorias para apoyar proyectos de investigación de ciencia aplicada y sería muy interesante analizar la participación de los diferentes tipos de instituciones en estas convocatorias.

Una pregunta importante es plantearnos si los apoyos a la investigación científica básica, han jugado algún papel en la descentralización de la ciencia en el país. Al separar a las instituciones que han recibido apoyos entre las que se encuentran en el D.F. y las que se encuentran en los estados de la República, se nota en primer lugar, que desde 2002 se apoyaron más proyectos para desarrollarse en los Estados (52\%), que en el Distrito Federal (48\%) y ha habido una tendencia a que se aumente la proporción de proyectos que se realizan en los Estados, de tal forma que entre 2009 y 2011, el 60\% de los proyectos se realizaron en los Estados (gráfica 4). Por otra parte, cuando analizamos esto para las IES públicas, vemos que las universidades estatales han tenido un incremento muy importante pasando de un 16.6\% en 2002 a un 33.4\% en 2011. Para 2010 y 2011 la proporción de proyectos que se aprobaron para las universidades estatales alcanzaron a ser prácticamente los mismos que se aprobaron para las IES del D.F.

Otro aspecto interesante es que, si se desagregan los proyectos por modalidad, se ve que el incremento en proyectos que se dio en las universidades estatales, se debe en primer lugar a la modalidad de profesor investigador. En 2002, del total de proyectos en esta modalidad, 14.6\% fueron otorgados a universidades estatales, mientras que en 2011 este valor casi se duplicó pasando a $28.1 \%$. Para la modalidad de grupos de investigación pasó de $14.3 \%$ en 2002 a $24.6 \%$ en 2011 y para investigador joven pasó de $31.7 \%$ en 2012 a $40.1 \%$ en 2011.

Desde 1996, la SEP tiene funcionando un Programa de Mejoramiento del Profesorado (PROMEP), mediante el cual se ha contratado una gran cantidad de profesores con doctorado para las universidades estatales, además de que se ha promovido el que los profesores que ya están contratados realicen estudios de doctorado. El PROMEP reporta que de 1996 a diciembre 
de 2011, logró incrementar el número de profesores con doctorado en las universidades estatales de 1,242 a 11,721 (PROMEP, 2013). Por otra parte, el PROMEP, a partir de 2001 otorga también apoyos para proyectos de investigación, los cuales, aunque se dan por un único año, seguramente han contribuido a fortalecer la capacidad de investigación de los investigadores participantes en dicho programa. El programa PROMEP pudiera ayudar a explicar el crecimiento tan importante que ha tenido la participación de las universidades estatales en los apoyos a investigación científica básica.

Por otra parte, el sistema tecnológico del la SEP, cuyas unidades se encuentran distribuidas por todos los estados de la República, ha tenido una participación muy baja en los apoyos a investigación científica básica. Aunque de 2006 a 2011 la participación se incrementó discretamente, no se define una tendencia a un incremento constante (gráfica 4). Es interesante que este sistema educativo no se incorporó al PROMEP, sino hasta 2008 (PROMEP, 2013). Es posible que los efectos del programa PROMEP, en cuanto a la participación en los proyectos de investigación científica básica, tarden algunos años en manifestarse y por eso no se alcance a ver todavía un efecto en el sistema tecnológico. Será interesante abordar este punto en los próximos años.

Otro aspecto a comentar, es que la participación porcentual de la producción mexicana de artículos científicos en revistas internacionales, ha pasado de 5,515 artículos en 2002 a 10,449 en 2011, un incremento del 90\% (Informe General del Estado de la Ciencia y la Tecnología, 2011). Dada la gran cantidad de proyectos de investigación científica básica que se han financiado en los diez últimos años, sería muy interesante realizar un estudio bibliométrico, para averiguar qué papel han jugado estos apoyos en este incremento de publicaciones científicas.

\section{Conclusiones}

Los resultados de este trabajo dan un panorama bastante claro de cuáles son las fortalezas en ciencia básica en México por área del conocimiento. Además de las áreas tradicionalmente asociadas a la ciencia básica (física, matemáticas, biología y química), hay fortalezas en áreas que por su orientación son más aplicativas (medicina, biotecnología e ingeniería).

Los apoyos a la investigación científica básica del CONACYT, han beneficiado en forma importante a los investigadores jóvenes y han promovido la descentralización de la investigación en el país, fortaleciéndola en particular en las universidades estatales. Este es un resultado importante 
del apoyo que ha dado CONACYT a la investigación científica básica en México y por lo tanto se recomienda que se mantenga.

Por otra parte, la mayoría de los apoyos se han otorgado a centros de investigación e instituciones públicas, jugando un papel pequeño las contrapartes privadas. Dentro de los centros de investigación e instituciones públicas, las instituciones de educación superior son las que tienen la mayor participación.

Todos estos resultados, se lograron mediante una política de apoyo con base a la calidad de los proyectos, juzgada por pares académicos, sin favorecer áreas de conocimiento o centros de investigación o instituciones en particular. La pregunta que queda por responder recae en la posibilidad de que pudieran obtener mejores resultados revisando y modificando esta política. Derivado del análisis presentado en este trabajo, preocupa el hecho de que no se hayan logrado promover efectivamente, ni los proyectos multidisciplinarios, ni los proyectos de grupos de investigación. Una posible recomendación es que se aumenten los techos financieros en esta área y modalidad, para hacerlos más atractivos.

El análisis de datos de los diez años pasados, que se presentan en este trabajo, podrá servir como base para revisar y de ser necesario replantear las políticas de apoyos a la investigación científica básica del CONACYT.

\section{Bibliografía}

Caraça J., Lundvall B- $\AA$ y Mendoça S., 2009, “The Changing Role of Science in the Innovation Process: From Queen to Cinderella?", Technological Forecasting \& Social Change, vol. 76, pp. 861-867.

CONACYT, 2012, Programa Institucional 2008-2012, México, CONACYT.

CONACYT, 2011, Informe General del Estado de la Ciencia y la Tecnología, México Consejo Nacional de Ciencia y Tecnología.

Convocatorias de Investigación Científica Básica, bases y términos de referencia desde la 2002 a la 2012, documentos electrónicos en: <http://www.conacyt. gob.mx/FondosyApoyos/Sectoriales/InvestigacionBasicaAplicada/SEP/Basica/Paginas/Convocatorias-y-Resultados.aspx> 
Informe de Labores del Consejo Nacional de Ciencia y Tecnología, 2012, documento electrónico en: <http://www.siicyt.gob.mx/siicyt/cms/paginas/Estadistica. jsp>.

Ley de Ciencia y Tecnología, 5 de junio de 2002, documento electrónico en: <http:/ / www.diputados.gob.mx/LeyesBiblio/ref/lct.htm>.

Ley Orgánica del Consejo Nacional de Ciencia y Tecnología, 5 de junio de 2002, documento electrónico en: <www.diputados.gob.mx/LeyesBiblio/pdf/243.pdf>.

Mansfield E, 1991, “Academic Research and Industrial Innovation”, Research Policy, vol. 20, pp. 1-12.

Márquez, M. T., 1982, 10 años del Consejo Nacional de Ciencia y Tecnología, México, CONACYT.

OCDE, 2012, La Estrategia de Innovación de la OCDE. Empezar hoy el Mañana, México, Foro Consultivo Científico y Tecnológico, AC.

PROMEP (Programa de Mejoramiento del Profesorado, Secretaría de Educación Pública), 2013, “Presentación”, en: <http://promep.sep.gob.mx/presentacion. html>.

Stephan, P., 2001, How Economics Shapes Science, Cambridge, Harvard University Press.

Recibido el 5 de febrero de 2013 Aceptado el 10 de octubre de 2013 